\title{
Einleitung: Konstruktionen des Publikums
}

\author{
Raphaela Knipp · Stephan Habscheid · Christine Hrncal
}

Online publiziert: 12. Oktober 2017

(C) Springer-Verlag GmbH Deutschland 2017

Bei dem vorliegenden Heft handelt es sich um den zweiten Teil einer Doppelausgabe der Zeitschrift für Literaturwissenschaft und Linguistik, die sich - anknüpfend an eine Siegener Tagung ${ }^{1}$ - dem thematischen Schwerpunkt >Publikum< widmet. Während sich das erste Heft mit den Alltagspraktiken des Publikums aus Sicht von Rezipientinnen und Rezipienten befasst (Ausgabe 4, 2016), hat es sich für unsere Überlegungen als ebenso zentral erwiesen, zu fragen, wie >Kunstschaffende< sowie die Wissenschaften >ihr< bzw. das Publikum konzeptualisieren und konstruieren. Der Fokus dieser zweiten Ausgabe des Themenschwerpunktes liegt daher auf den Konstruktionen des Publikums. Derartige Konstruktionen sind teilweise - explizit

\footnotetext{
${ }^{1}$ Die beiden Themenhefte gehen auf die interdisziplinäre Tagung »Alltagspraktiken des Publikums: Theater, Literatur, Kunst, Populärkultur« zurück, die am 29. Februar und 1. März 2016 im Apollo-Theater Siegen stattfand. Die Tagung wiederum basierte auf einer Kooperation des DFG-Projektes »Theater im Gespräch. Sprachliche Kunstaneignungspraktiken in der Theaterpause« unter der Leitung von Stephan Habscheid (Universität Siegen) und Erika Linz (Universität Bonn), des durch das Siegener DFG-Graduiertenkolleg »Locating Media« geförderten Projektes »Begehbare Literatur. Eine literatur- und kulturwissenschaftliche Studie zum Literaturtourismus « von Raphaela Knipp sowie des Handbuch-Projektes »Sprache in der Kunstkommunikation « unter der Leitung von Marcus Müller (TU Darmstadt) und Heiko Hausendorf (Universität Zürich). Zum Konzept der Tagung sowie den theoretischen Grundlagen siehe auch die Einleitung zum ersten Heft Alltagspraktiken des Publikums: Theater, Literatur, Kunst, Populärkultur, Zeitschrift für Literaturwissenschaft und Linguistik 4 (2016), S. 463-468.
}

\section{R. Knipp}

Ruhr-Universität Bochum, 44810 Bochum, Deutschland

E-Mail: raphaela.knipp@rub.de

S. Habscheid $(\square) \cdot$ C. Hrncal

Universität Siegen, 57068 Siegen, Deutschland

E-Mail: habscheid@germanistik.uni-siegen.de

C. Hrncal

E-Mail: hrncal@germanistik.uni-siegen.de 
oder als interaktiv vorausgesetzter Verstehenshintergrund - in alltägliche kommunikative Praktiken der ästhetischen Gestaltung eingebettet, zum Teil sind sie aber auch Gegenstand von - ihrerseits öffentlichen - Debatten und Reflexionen, die von der unmittelbaren Bearbeitung praktischer Aufgaben entbunden sind: in der Wissenschaft und Publizistik, der ästhetischen Theorie und nicht zuletzt der Kunst/Literatur selbst. Soweit die Konzepte an sprachliche Ausdrücke gebunden und durch sie (mit) strukturiert sind, reicht der Gegenstandsbereich in die Semantik und Grammatik des Sprechens über das Publikum hinein, die sich bereichsspezifisch mit anderen Modalitäten der Darstellung (z.B. Gestik in Theaterproben, statistische Quantifizierung in Befragungsstudien) verbinden kann.

Mehr darüber zu wissen, wer eigentlich >das Publikum< ist, wie es sich zusammensetzt und was es >begehrt< (Hennion/Méadel 2013), ist vermutlich ein Interesse vieler Kunst- und Kulturschaffender sowie jener Akteure, die mit der Distribution und Vermittlung ästhetischer Artefakte betraut sind. Dies belegen allein schon die zahlreichen Maßnahmen und Formate, die entwickelt wurden, um das Publikum von außen betrachtet zu vermessen, vielleicht auch dem Publikum kulturell >näher< zu kommen (für eine kulturökonomische Perspektive auf Instrumente der Publikumsforschung vgl. etwa Glogner-Pilz 2012; ferner z.B. Sutter 2016; Reussner 2010): Dazu zählen etwa die Erfassung von Einschaltquoten im Bereich von Film und Fernsehen, Lesererhebungen im Printbereich, Besucherbefragungen und Gästebücher in Kunst- und Literaturausstellungen sowie daraus resultierende Besucherund Ausstellungstypen, Umfragen unter Theaterbesucherinnen und -besuchern, Beteiligungen des Publikums, z.B. in Form von Zuschaueranrufen in Talkshows oder Leserbriefen, sowie nicht zuletzt, im digitalen Zeitalter, die Aktivierung des Publikums über Social-Media-Kanäle - die Liste der Beispiele ließe sich zweifelsohne weiterführen. Letztlich aber müssen all diese Versuche, des Publikums habhaft zu werden, zumindest in Teilen eine Aporie bleiben, insofern das Publikum immer auch eine idiosynkratische, empirisch kaum bzw. nur schwer zu überblickende Größe ist (Kammerer 2012; Schenk/Tröhler/Zimmermann 2010; Ang 2001). Wenn daher also die Rede > vom Publikum< ist, so muss dieses stets auch im Fluchtpunkt bestimmter Konstruktionen, Heuristiken, Typisierungen etc. betrachtet und situiert werden, welche die >positivistische Leerstelle< gewissermaßen modellhaft oder idealtypisch zu besetzen - und ggf. auf dieser Basis das Publikum empirisch zu ergründen versuchen. In diesem Sinn thematisiert das vorliegende Heft auch einen blinden Fleck, der in der Erforschung der Alltagspraktiken des Publikums noch weitgehend außerhalb des Blickfeldes blieb.

Dementsprechend fragen die Beiträge dieses Heftes, wie Kunst- und Kulturschaffende (z.B. Theatermachende, Autorinnen und Autoren oder Medienproduzenten), aber auch Kulturjournalisten und nicht zuletzt Wissenschaftlerinnen und Wissenschaftler das Publikum konstruieren: d.h. im Einzelnen, wie sie es beobachten und beschreiben, antizipieren und adressieren, semantisieren und inszenieren sowie konzeptualisieren und theoretisieren. Welche Rolle schließlich weisen sie dem Publikum dabei zu und wie beteiligen sie es darin an Kunst?

Diese Fragen nach den Konstruktionen des Publikums lassen sich wiederum in mindestens drei Ebenen gliedern, die in den folgenden Beiträgen mit unterschiedlicher Schwerpunktsetzung thematisch werden: 
- Erstens, ließe sich das Publikum im Fluchtpunkt theoretisch-historischer (versprachlichter) Konzeptualisierungen betrachten: Darunter fallen zum einen historische Manifestierungen und Äußerungen über das Publikum und seine Rolle (s. dazu insbesondere Bosse in diesem Heft), wie sie sich u.a. etwa in Lessings Hamburgischer Dramaturgie (zwischen 1767 und 1769) oder in Brechts Ausführungen über Das epische Theater (um 1936 verfasst, vgl. Brecht 1963) finden, um nur zwei prominente Beispiele für historisch-ästhetische Abhandlungen zu nennen, in denen auf das Publikum idealtypisch Bezug genommen wird. Zum anderen fallen hierunter all jene theoretischen und wissenshistorischen Klassifizierungen und Bestimmungen des Publikums in den verschiedenen wissenschaftlichen Disziplinen wie etwa der Soziologie, der Sprach- und Literaturwissenschaften, der Philosophie, der Kulturwissenschaften (insbesondere der Cultural Studies) sowie nicht zuletzt der Wirtschaftswissenschaften, in denen sich jeweils eigene, fächerspezifische Publikumskonstrukte herausgebildet und etabliert haben. ${ }^{2}$

- Zweitens, kann die Frage nach Publikumskonstruktionen auf einer formal-ästhetischen Ebene gestellt werden: Dahinter verbirgt sich die Annahme, dass das Publikum bereits immer schon in der formal-ästhetischen bzw. inhaltlichen Gestaltung, Konzipierung oder Darstellung künstlerischer Artefakte (seien dies Filme, literarische Texte, Theaterstücke oder Ausstellungen etc.) konzeptuell verankert bzw. >mitgedacht< ist (s. dazu Moshövel/Sahm und Schmidt in diesem Heft). Prominent zu nennen sind diesbezüglich beispielsweise die Ansätze der so bezeichneten Rezeptionsästhetik, die das Publikum auf die Rolle »impliziter « Leser/innen (Iser 1972) festlegt, deren Rezeptionstätigkeit durch den jeweiligen Text gewissermaßen vorgegeben ist und somit durch diesen konstruiert wird. Dies sei hier nur an einem kurzen Beispiel veranschaulicht, nämlich der paratextuellen Kategorie $>$ Genre $<$ : Ob ein literarischer Text z.B. als >Kriminalroman $<$, >historischer Roman $<$ oder etwa >Fantasyroman< ausgewiesen wird, beruht auf bestimmten ästhetischen Konventionen sowie daran gebundener (antizipierter) Publikumserwartungen; gleiches gilt auch für Genres im Bereich von Film und Fernsehen etc. ${ }^{3}$

- Drittens schließlich lassen sich Publikumskonstruktionen als Ergebnis bestimmter gesellschaftlicher und/oder institutioneller Diskurse verfolgen und untersuchen (s. dazu Fix und Hills in diesem Heft): Welche gesellschaftliche Funktion hat Kunst in Bezug auf das Publikum zu erfüllen (z.B. Bildung, Erziehung, Aufklärung oder Ermunterung, Unterhaltung etc.), bzw. inwiefern werden solche Funktionen und

\footnotetext{
2 In diesem Zusammenhang ist erstens auffallend, dass wissenschaftliche Publikumskonstrukte meist in Abhängigkeit zu bestimmten Einzelmedien stehen (die >Zuschauer/innen< und >Zuhörer/innen< im Bereich von Film und Fernsehen, die >Leser/innen< im Bereich von Literatur und Printmedien sowie die >User/ innen< oder gar >Prosumer/innen< im Umfeld digitaler Medien). Dies ist jedoch insofern problematisch, als dabei die Frage nach den intermedialen Bezügen des Publikums bzw. zwischen Publika unbeantwortet bleibt (z.B. Leser/innen, die gleichzeitig Filme rezipieren und/oder weitere Medienangebote konsumieren; s. zu dieser Problematik auch die frühen Überlegungen von Radway 1988). Zweitens bleibt die wissenschaftliche Auseinandersetzung mit dem Publikum selbst dort, wo sie ihren empirischen Anspruch behauptet, auf Konstrukte angewiesen (s. z.B. die frühen Arbeiten der Cultural Studies, die ihrer empirischen Forschung u.a. Stanley Fishs (1980) Konzept der »interpretive community« zugrunde legen; für einen Überblick hierzu s. Lingenberg 2015).

$3 \mathrm{Zu}$ ästhetischen Konventionen und darauf beruhenden bzw. antizipierten Erwartungshorizonten von Publika s. insbesondere Jauß 1979.
} 
Aufgaben je nach Kunstform und Publikum unterschiedlich gewichtet und zugeordnet - z.B. das Kino als Ort der Unterhaltung, das Theater hingegen als Ort der Bildung und Aufklärung und so fort? Welches Publikum wünschen sich bestimmte Institutionen (s. den Beitrag von Fix) und welche Verhaltensweisen und Reaktionen seitens des Publikums werden diesbezüglich als erwartbar bzw. als adäquat vorausgesetzt (s. den Beitrag von Hills)?

Auf der Basis dieser Überlegungen kann als Arbeitsauftrag abgeleitet werden, jene Theoreme, jene formal-ästhetischen und inhaltlichen Gestaltungsmerkmale sowie jene (historischen) Sprechweisen und Handlungen zu identifizieren und zu analysieren, die implizit oder explizit auf Konstruktionen des Publikums sowie damit einhergehende Rollenverständnisse verweisen. Dieser Aufgabe gehen die folgenden Beiträge nach, indem sie Publikumskonstruktionen z.B. im Rahmen von Theaterproben, in Interviews von Theaterintendanten, im Kontext wissenschaftlicher Debatten und historischer Öffentlichkeiten sowie nicht zuletzt in literarischen Texten nachspüren.

In einem diskursanalytischen Zugriff untersucht der Beitrag von Ulla Fix, wie zwei Intendanten des Leipziger Schauspielhauses ihr Publikum sprachlich-medial konstruieren. Deutlich werden dabei einerseits zwei sich grundlegend voneinander unterscheidende Auffassungen darüber, welche gesellschaftliche Bedeutung dem Theater und Theaterpublikum im gegenwärtigen Kulturbetrieb zuzuweisen ist. Zum anderen weist der Beitrag das Publikum als soziale Konstruktion aus, in der einerseits bestimmte Erwartungen seitens des Publikums und andererseits Wunschvorstellungen eines >idealen< Publikums seitens der Theatermachenden zusammenfallen bzw. vielmehr >auseinanderfallen $<$.

Auch Axel Schmidt fokussiert das Theater, jedoch in der alltagspraktisch situierten, intimen und öffentlichkeitsabgeschirmten Situation der Theaterprobe. Dabei zeigt Schmidt, wie das Publikum als antizipierte Größe in den Theaterproben eine Rolle spielt, indem sich die Theatermachenden sprachlich und körperlich auf es beziehen. Konkret fragt Schmidt, welche Annahmen und Konstruktionen des Publikums in diesen Bezugnahmen beobachtbar sind. Die Theaterprobe wird dabei letztlich als fiktionale Kommunikationssituation zwischen Theatermachenden und Publikum ausgewiesen, in der »imaginäre Publikumsbezüge « interaktiv realisiert werden, um bestimmte Darstellungsweisen zu plausibilisieren.

Der Beitrag von Matt Hills befasst sich mit Konstruktionen und Stereotypisierungen von >Fans $<$ und $>$ Fantum $<$ in medialen sowie insbesondere theaterbezogenen Diskursen. Hills' Ausgangsbeobachtung ist, dass die so bezeichneten Fan Studies ${ }^{4}$ das Theater als Ort des >Fan-Seins< bislang eklatant vernachlässigt haben, sowie Studien über das Theater häufig den Aspekt des Publikums (insbesondere den der >Theaterfans $<$ ) ausblenden. Den Grund dafür lokalisiert Hills in der traditionellen Abgrenzung des Theaters (wie im Übrigen auch der Literatur, bildenden Kunst

\footnotetext{
4 Die sogenannten, vorwiegend im anglo-amerikanischen Sprachraum verorteten Fan Studies haben sich aus den Cultural- und Audience Studies der 1980er und 1990er-Jahre formiert. Ihr Anliegen ist u.a. eine kritische Auseinandersetzung mit stereotypen Konzeptualisierungen von >Fan $<$ und >Fantum $<$, die zudem häufig bestimmten, populären Medien zugerechnet werden.
} 
oder der Oper) als >hochkulturelle<, >anspruchsvolle $<$ Kunstform, die keinen Platz für >Fan-Sein < lässt, von populärkulturellen Formen (z.B. Film und Fernsehen), in deren Kontext Fanpraktiken als legitim erachtet werden. Unter Rückgriff auf u.a. Bourdieus Feldtheorie unterzieht der Beitrag diese Opposition einer kritischen Reflexion und zeigt, wie das Theater, insbesondere in der jüngeren Gegenwart, immer auch - mehr oder weniger gewollt - mit populärkulturellen Formen und Praktiken interagiert.

Neben diesen gegenwartsbezogenen Beiträgen soll in diesem Heft insbesondere auch die historische Dimension der Publikumskonstruktionen Berücksichtigung finden. Aus historischer Perspektive stellen sich spezifische, u.a. vor allem methodische Herausforderungen, insofern die historisierende (Re-)Konstruktion von Publika stets auf historische Dokumente (z.B. Autobiographien, Briefwechsel, Lesetagebücher, Nachlassinventare, Buchbesitz etc.) angewiesen ist, die mitunter ihrerseits wiederum konstruktiven Charakter besitzen. Dies ist ein wichtiger Aspekt, dem unter anderem Heinrich Bosse in seinem für eine Perspektive auf historische (literarische) Öffentlichkeiten grundlegenden Beitrag zum Strukturwandel des Publikums im 18. Jahrhundert nachgeht. Bosses zentrale These, die er anhand reichhaltigen historischen Materials entfaltet, lautet, dass sich das Publikum im 18. Jahrhundert von den Mitgliedern der res publica literaria (»Republik der Gelehrten «) zu den Angehörigen der deutschen Nation wandelt mit Folgen für den Büchermarkt.

Der zweite historisch orientierte Beitrag von Andrea Moshövel und Heike Sahm fragt anhand von zwei cross-dressing-Erzählungen des Mittelalters und der frühen Neuzeit nach der Funktion und Urteilskraft vormoderner Öffentlichkeit sowie ihrer literarischen Inszenierung. Mittels eines Close Reading der Texte zeigen die Autorinnen auf, wie in beiden Erzählungen das Motiv des cross-dressing mit der frühneuzeitlichen Diskussion von Öffentlichkeit verknüpft wird, wobei die Texte die Instanz der Öffentlichkeit jeweils unterschiedlich konzipieren und bewerten: im einen Fall als weitgehend unzuverlässige Größe, im anderen Fall als normsetzende und -bewahrende Größe. Damit lassen sich beide Textbeispiele schließlich als Problematisierung der frühneuzeitlichen literarischen Debatte über die Verlässlichkeit und Reichweite der öffentlichen Meinung lesen.

\section{Literatur}

Ang, Ien: »Zuschauer, verzweifelt gesucht«. In: Ralf Adelmann u.a. (Hg.): Grundlagentexte zur Fernsehwissenschaft. Theorie, Geschichte, Analyse. Konstanz 2001, S. 454-483.

Brecht, Bertolt: »Das epische Theater«. In: Ders.: Schriften zum Theater 3. Frankfurt a.M. 1963.

Fish, Stanley: Is There a Text in this Class? The Authority of Interpretive Communities. Cambridge/London 1980.

Glogner-Pilz, Patrick: Publikumsforschung. Grundlagen und Methoden. Wiesbaden 2012.

Habscheid, Stephan/Hrncal, Christine/Knipp, Raphaela/Linz, Erika: »Einleitung: Alltagspraktiken des Publikums«. In: Alltagspraktiken des Publikums: Theater, Literatur, Kunst, Populärkultur, Zeitschrift für Literaturwissenschaft und Linguistik 4 (2016), S. 463-468.

Hennion, Antoine/Méadel, Cécile: »In den Laboratorien des Begehrens: die Arbeit der Werbeleute«. In: Tristan Thielmann/Erhard Schüttpelz/Peter Gendolla (Hg.): Akteur-Medien-Theorie. Bielefeld 2013, S. 341-376.

Iser, Wolfgang: Der implizite Leser. Kommunikationsformen des Romans von Bunyan bis Beckett. München 1972. 
Jauß, Hans Robert: »Literaturgeschichte als Provokation der Literaturwissenschaft «. In: Rainer Warning (Hg.): Rezeptionsästhetik. 2. Aufl. München 1979, S. 126-162.

Kammerer, Dietmar: »Vorwort. Vom Publicum«. In: Ders. (Hg.): Vom Publicum. Das Öffentliche in der Kunst. Bielefeld 2012, S. 7-11.

Lessing, Gotthold Ephraim: Hamburgische Dramaturgie. 2 Bde. Hamburg/Bremen 1767 und 1769.

Lingenberg, Swantje: »Publika und Interpretationsgemeinschaften«. In: Andreas Hepp u.a. (Hg.): Handbuch Cultural Studies und Medienanalyse. Wiesbaden 2015, S. 137-146.

Radway, Janice: »Reception Study: Ethnography and the Problems of Dispersed Audiences and Nomadic Subjects«. In: Cultural Studies 2, 3 (1988), S. 359-376.

Reussner, Eva M.: Publikumsforschung für Museen. Internationale Erfolgsbeispiele. Bielefeld 2010.

Schenk, Irmbert/Tröhler, Margrit/Zimmermann, Yvonne: »Vom idealen Zuschauer zur sozialen Praxis der Rezeption.«In: Dies. (Hg.): Film, Kino, Zuschauer. Filmrezeption. Marburg 2010, S. 9-16.

Sutter, Tilmann: »Massenmediale Inklusionsprozesse. Adressierung, Einbeziehung und Beteiligung des Publikums im Fernsehen«. In: Zeitschrift für Theoretische Soziologie 5, 2 (2016), S. 182-213. 